\title{
Sodium butyrate and dexamethasone promote exocrine pancreatic gene expression in mouse embryonic stem cells
}

\author{
Meng REN ${ }^{1}$, Li YAN${ }^{1}$, Chang-zhen SHANG ${ }^{2}$, Jun $\mathrm{CAO}^{2}$, Fang-ping $\mathrm{LI}^{1}$, Jing-yi $\mathrm{LI}^{1}$, Hua CHENG ${ }^{1, *}$, Jun MIN², \\ ${ }^{1}$ Departments of Endocrinology and ${ }^{2}$ Hepatobiliary Surgery, The Second Affiliated Hospital of Sun Yat-sen University, Guangzhou \\ 510120, China
}

\begin{abstract}
Aim: The feasibility of inducing endocrine pancreatic differentiation of embryonic stem (ES) cells has been well documented. However, whether ES cells possess the potential for exocrine pancreatic differentiation requires further exploration. Here, we investigated whether sodium butyrate and glucocorticoids were conducive to the exocrine pancreatic differentiation of ES cells.

Methods: E14 mouse ES cells were cultured in suspension to form embryoid bodies (EBs). These EBs were cultured in differentiating medium containing varying concentrations of sodium butyrate. The effects of activinA and dexamethasone (Dex) on exocrine differentiation were also explored. Finally, the combination of sodium butyrate, activinA, and Dex was used to promote the differentiation of exocrine pancreatic cells. Specific exocrine pancreatic gene expression was detected by reverse transcription polymerase chain reaction (RT-PCR) and amylase expression was examined by immunofluorescence staining. Flow cytometry analysis was also performed to determine the percentage of amylase-positive cells after the treatment with activinA, sodium butyrate, and Dex.

Results: Exposure of ES cells to $1 \mathrm{mmol} / \mathrm{L}$ sodium butyrate for 5 days promoted exocrine pancreatic gene expression. Further combination with Dex and other pancreatic-inducing factors, such as activinA, significantly enhanced the mRNA and protein levels of exocrine pancreatic markers. Additionally, flow cytometry revealed that approximately $17 \%$ of the final differentiated cells were amylase-positive. Conclusion: These data indicate that the exocrine pancreatic differentiation of ES cells can be induced by activinA, sodium butyrate, and Dex, providing a potential tool for studying pancreatic differentiation and pancreas-related diseases.
\end{abstract}

Keywords: embryonic stem cells; sodium butyrate; dexamethasone; differentiation; exocrine pancreatic cells

Acta Pharmacologica Sinica (2009) 30: 1289-1296; doi: 10.1038/aps.2009.115; published online 24 August 2009

\section{Introduction}

Mouse embryonic stem (ES) cells are isolated from the inner cell mass of blastocysts, which are capable of indefinite and pluripotential proliferation ${ }^{[1,2]}$. Certain differentiation factors involved in embryonic development can differentiate ES cells into cell types from all three germ layers in vitro ${ }^{[3-5]}$. More specifically, recent studies have demonstrated the capacity of ES cells to differentiate into endocrine pancreatic or insulin-producing cells in vitro ${ }^{[6-8]}$. However, the pancreas is composed of both exocrine and endocrine compartments, and although ES-derived pancreatic $\beta$-cell differentiation has been characterized, little is known regarding exocrine pancreatic differentiation.

Sodium butyrate is a histone deacetylase inhibitor reported

\footnotetext{
* To whom correspondence should be addressed.

E-mail drjunmin@yahoo.com.cn (Jun MIN); bear3151@yahoo.cn (Hua CHENG)

Received 2009-03-31 Accepted 2009-06-30
}

to promote the differentiation of many cell types through chromatin rearrangement and histone deacetylation ${ }^{[9-11]}$. Recent research demonstrated that sodium butyrate can activate early pancreatic development genes in ES cells ${ }^{[12]}$ and induce ES cells to differentiate into islet-like clusters ${ }^{[8]}$. Our previous work has also suggested that sodium butyrate can induce endoderm differentiation. In the present study, we investigated whether exocrine pancreatic cells could be generated from ES cells upon exposure to sodium butyrate. Our results revealed that treatment with $1 \mathrm{mmol} / \mathrm{L}$ sodium butyrate for 5 days was conducive to induce exocrine pancreatic differentiation.

Some studies have shown that glucocorticoids influence exocrine pancreas differentiation and increase exocrine gene expression. However, whether glucocorticoids affect the exocrine differentiation of ES cells remains uncertain. In the current study, we found that dexamethasone (Dex) promoted marked exocrine gene expression and the combination of activinA, sodium butyrate, and Dex significantly increased 
the mRNA and protein levels of exocrine markers. These data may be useful in the development of new strategies to direct ES-cell differentiation toward the exocrine pancreatic lineage in vitro. Thus, these findings provide a novel foundation for research on pancreatic differentiation and disease.

\section{Materials and methods Cell culture}

Undifferentiated E14 mouse ES cells (ATCC, Manassas, VA, USA) were maintained on gelatin-coated dishes in Dulbecco's modified Eagle's medium (DMEM; GIBCO, Grand Island, NY, USA) supplemented with 15\% fetal bovine serum (Hyclone, Rockville, MD, USA), $1000 \mathrm{U} / \mathrm{mL}$ recombinant mouse leukemia inhibitory factor (LIF; Chemicon, Temecula, CA, USA), $1 \%$ non-essential amino acids, $1 \mathrm{mmol} / \mathrm{L}$ glutamine, and 0.1 mmol/L $\beta$-mercaptoethanol (Sigma-Aldrich, MO, USA). The above culture medium, excluding LIF, was defined as the differentiating medium.

\section{In vitro differentiation of ES cells}

To induce differentiation, ES cells were trypsinized and resuspended in differentiating medium to form embryoid bodies (EBs). Four days later, the EBs were transferred into 6-well plates coated with $0.1 \%$ gelatin and cultured in differentiating medium in the presence of varying sodium butyrate concentrations. In some experiments, $25 \mathrm{ng} / \mathrm{mL}$ activinA (R\&D Systems Inc, Minneapolis, MN, USA) and/or $1 \times 10^{-7} \mathrm{~mol} / \mathrm{L}$ dexamethasone (Dex; Sigma-Aldrich) was added to further stimulate differentiation.

\section{Fluorescent-activated cell sorting (FACS)}

To quantify the survival rate of ES cells after exposure to sodium butyrate, annexin $\mathrm{V}$, and propidium iodide (PI) staining was performed, followed by flow cytometry. Briefly, cells were exposed to different concentrations of sodium butyrate, ranging from 1 to $10 \mathrm{mmol} / \mathrm{L}$, for $48 \mathrm{~h}$. The cells were then harvested, washed twice with cold phosphate-buffered saline (PBS), and subjected to annexin V and PI staining. After staining, the samples were analyzed by FACS (Becton Dickinson) using CellQuest Research Software (Becton Dickinson).

\section{Reverse transcription polymerase chain reaction (RT-PCR)}

Differentiated cells were collected at different time points for analysis of the mRNA expression of exocrine pancreatic genes. First-strand cDNA was generated using a commercial TaKaRa RT kit (TaKaRa, Japan) and primer sequences used in PCR are provided in Table 1. All quantifications were performed using mouse $\beta$-actin as an internal standard. PCR was conducted with the following thermal profiles: denaturation at $94{ }^{\circ} \mathrm{C}$ for 5 min, followed by 30-35 cycles of $30 \mathrm{~s}$ at $94^{\circ} \mathrm{C}, 45 \mathrm{~s}$ at optimal annealing temperatures (as described in Table 1), and $45 \mathrm{~s}$ at $72{ }^{\circ} \mathrm{C}$. The program was finished by a 10 -min extension at $72{ }^{\circ} \mathrm{C}$. The amplified products were subjected to electrophoresis using $20 \mathrm{~g} / \mathrm{L}$ agarose gels and stained with ethidium

Table 1. Sequence information on the primers used for RT-PCR.

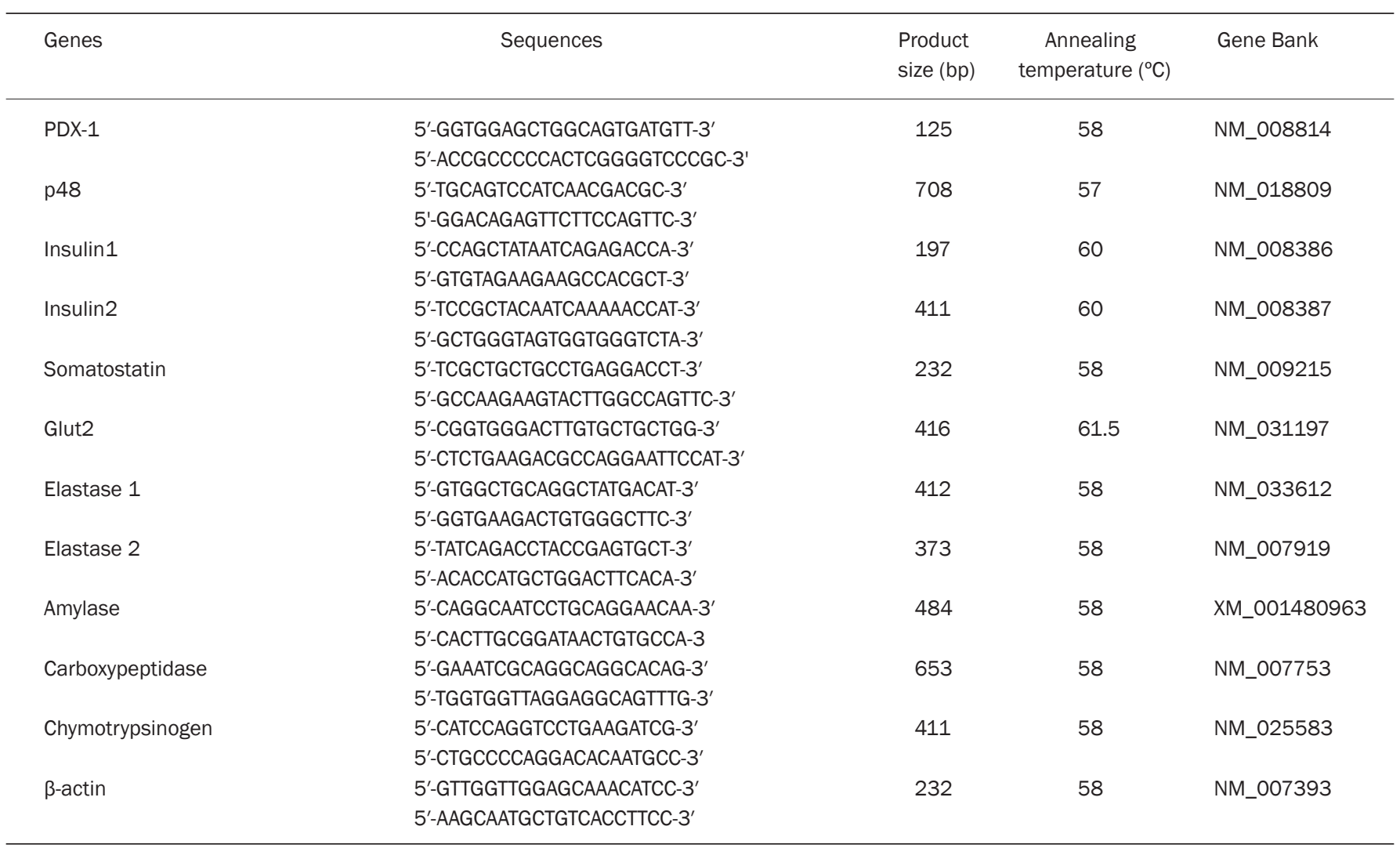


bromide. The ratios of exocrine markers to $\beta$-actin were then calculated. Semi-quantitative analysis of PCR products was performed using an Alpha Imager ${ }^{\text {TM }} 2000$ instrument (Alpha Innotech, San Leandro, CA, USA).

\section{Immunofluorescence}

Differentiated cells were washed with PBS, permeabilized with $0.2 \%$ Triton X-100 and blocked for 2-4 h at room temperature. Rabbit anti-amylase antibody (Sigma-Aldrich) was then applied overnight at $4{ }^{\circ} \mathrm{C}$. The next day, cells were washed with PBS three times for 15 min, treated with secondary antibody for $1 \mathrm{~h}$ and washed as before. Nuclei were stained blue with Hoechst 33258 (Sigma-Aldrich). The resultant immunofluorescence was viewed using a Nikon fluorescent microscope.

\section{Flow cytometry analysis}

Differentiated cells at the final stage of differentiation were dissociated using $0.25 \%$ trypsin-EDTA for 10-15 min, followed by centrifugation, washing, and fixation in $2 \%$ paraformaldehyde in PBS at room temperature for $15 \mathrm{~min}$. The cells were then permeabilized with IntraPrep permeabilization reagent (Invitrogen, CA, USA) for $30 \mathrm{~min}$. Next, the cells were incubated with $0.2 \mu \mathrm{g}$ amylase antibody (Sigma-Aldrich), washed with PBS, and stained with an appropriate secondary antibody conjugated to Alexa Fluor 488 at room temperature for 15 min. Finally, the cells were washed, resuspended in a final volume of $100 \mu \mathrm{L}$ PBS, and analyzed by FACS using CellQuest Research Software. Spontaneously differentiated cells were used as a control.

\section{Statistical analysis}

Each experiment was performed at least three times. All values are given as means $\pm S D$. Data were analyzed by oneway analysis of variance (ANOVA) using SPSS 10.0 software. $P<0.05$ was interpreted as a statistically significant difference.

\section{Results}

Expression of specific pancreatic markers during spontaneous ES cell differentiation

To study the expression of pancreatic markers in spontaneously differentiated cells, EBs were cultured in differentiating medium for 7 to 21 days. On d 7, 14, and 21, cells were collected and the expression of exocrine and endocrine markers was examined by semi-quantitative RT-PCR. As shown in Figure 1, both pancreatic/duodenal homeobox-1 (PDX-1), an important transcription factor involved in endocrine and exocrine gene activation ${ }^{[13]}$, and $\mathrm{p} 48$, the only factor known to be specifically required for the ontogeny of the exocrine pancreas $^{[14]}$, were detected on $\mathrm{d} 7$ of cell differentiation. On d 14, a small increase in the expression of these factors was noted, and the level increased gradually at later time points.

In the developing mouse pancreas, carboxypeptidase mRNA is the earliest enzyme transcript detected ${ }^{[15]}$. Here, we found that carboxypeptidase was expressed on $\mathrm{d} 7$ of cell differentiation, followed by a decrease in expression on $\mathrm{d} 14$ and 21 .

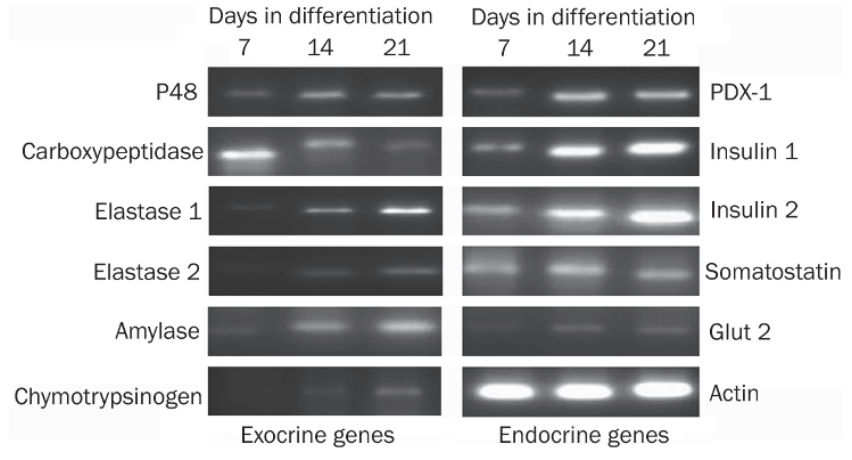

Figure 1. Expression of exocrine and endocrine pancreatic markers in spontaneously differentiated ES cells. EBs were formed in suspension culture for $4 \mathrm{~d}$ and then transferred into gelatin-coated dishes and grown in differentiating medium for $21 \mathrm{~d}$. On d 7, 14, and 21, cells were collected and the gene expression of exocrine (left image) and endocrine (right image) markers was measured by RT-PCR. $\beta$-actin was considered a control of equal RNA amount. This experiment was performed in triplicate.

Other exocrine markers, such as amylase, chymotrypsinogen, and elastases 1 and 2, were also detected on $\mathrm{d} 7$ and maintained constant levels at later time points. Moreover, spontaneously differentiated cells also showed a marked increase in gene expression of insulin 1 and 2, which increased continuously until d 21. The mRNA expression of somatostatin also increased from d 7 to $\mathrm{d} 14$ but decreased at later time points. Gene expression of glucose transporter-2 (Glut2), which plays an important role in glucose-stimulated insulin secretion, was also detected on $\mathrm{d} 7$ and exhibited an increase over time. Taken together, these data demonstrate that both exocrine and endocrine markers were expressed during spontaneous ES cell differentiation.

\section{Influence of different concentrations of sodium butyrate on} growth activities of ES cells

Sodium butyrate is known to promote endoderm cell differentiation as well as inhibit cell proliferation. Thus, the optimal concentration of sodium butyrate required to stimulate ES cell differentiation was studied first. After four days of EB formation using the suspension culture method, EBs were transferred into 6-well plates coated with $0.1 \%$ gelatin and cultured in differentiating medium in the presence of varying sodium butyrate concentrations $(1,3,5,7$, or $10 \mathrm{mmol} / \mathrm{L})$ for $48 \mathrm{~h}$. FACS was then used to evaluate EB growth activity. The results indicated that differentiated ES cells exposed to 1 to $3 \mathrm{mmol} / \mathrm{L}$ sodium butyrate exhibited higher survival rates $(82.75 \% \pm 6.7 \%$ at $1 \mathrm{mmol} / \mathrm{L}$ and $67.5 \% \pm 5.5 \%$ at $3 \mathrm{mmol} / \mathrm{L})$, whereas supplementation with 5 to $10 \mathrm{mmol} / \mathrm{L}$ sodium butyrate resulted in obvious cell apoptosis $(P<0.05)$. The cells' survival rate decreased to $45.51 \% \pm 3.2 \%, 5.85 \% \pm 1.9 \%$, and $2.89 \% \pm 0.2 \%$ when treated with 5,7 , and $10 \mathrm{mmol} / \mathrm{L}$ sodium butyrate, respectively (Figure 2). These data indicate that exposure to 1 to $3 \mathrm{mmol} / \mathrm{L}$ sodium butyrate was suitable for the survival and differentiation of ES cells. 

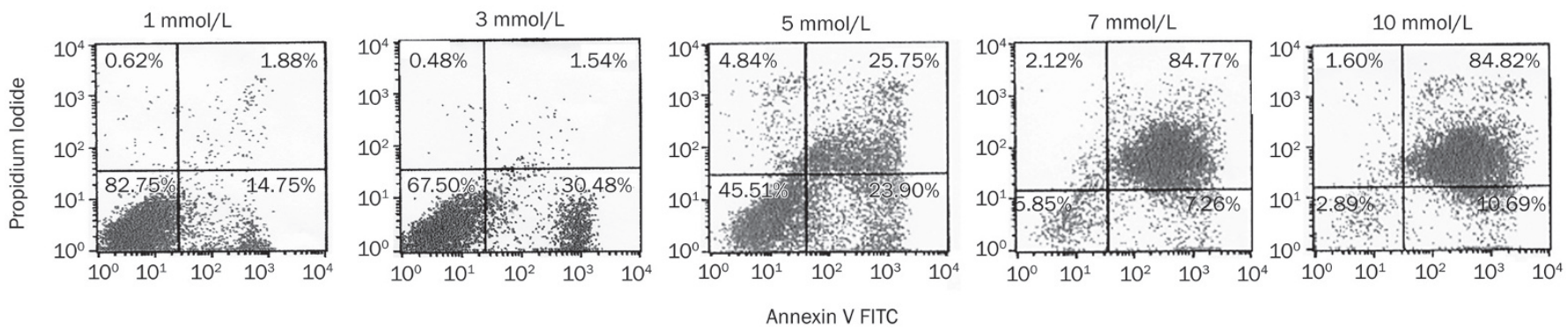

Figure 2. Effects of sodium butyrate at different concentrations on ES cell growth activities. Cells were exposed to different concentrations of sodium butyrate $(1,3,5,7$, or $10 \mathrm{mmol} / \mathrm{L})$ for $48 \mathrm{~h}$. Annexin $\mathrm{V}$ and PI staining was performed, followed by flow cytometry to detect cell apoptosis. The dot plot diagrams represent typical apoptotic and necrotic cell populations, as detected by Annexin $\mathrm{V}$ and PI staining. Values are given as means \pm SD. A representative example of the experiment, which was repeated three times, is shown.

\section{Sodium butyrate activates exocrine pancreatic differentiation of ES cells}

To investigate the effects of sodium butyrate on exocrine pancreatic differentiation, EBs were cultured in differentiating medium supplemented with 1,2 , or $3 \mathrm{mmol} / \mathrm{L}$ sodium butyrate for $5 \mathrm{~d}$. RT-PCR was then performed to analyze exocrine pancreatic gene expression. As shown in Figure $3 \mathrm{~A}$, sodium butyrate upregulated the expression of exocrine pancreatic markers, including amylase, elastases 1 and 2, carboxypeptidaseA, and chymotrypsinogen. A maximal effect was observed in EBs treated with $1 \mathrm{mmol} / \mathrm{L}$ sodium butyrate, whereas increasing sodium butyrate concentrations led to weaker gene expression of exocrine markers.

To study the effect of exposure time of sodium butyrate on cell differentiation, EBs were treated with $1 \mathrm{mmol} / \mathrm{L}$ sodium butyrate for 1 week. On d 1, 3, 5, and 7 of ES differentiation, cells were collected for analysis of the mRNA expression of exocrine pancreatic genes. After initial exposure to sodium butyrate, all pancreatic exocrine markers except amylase began to be expressed on $\mathrm{d} 3$ and peaked on $\mathrm{d} 5$. Then a slight decrease in exocrine gene expression was noted on $\mathrm{d} 7$. Meanwhile, amylase expression was detected on day 1 , peaked on d 3 and decreased slightly on d 5 (Figure 3B).

\section{Induction of exocrine pancreatic differentiation by activinA or Dex}

Transforming growth factor- $\beta$ (TGF- $\beta$ ) is a major regulator of exocrine and endocrine pancreatic cell fate. ActivinA, a member of the TGF- $\beta$ superfamily, has been reported to induce endoderm formation and regulate the early stages of pancreatic development ${ }^{[16]}$. In the present study, the effect of activinA on exocrine pancreatic gene expression was charac-

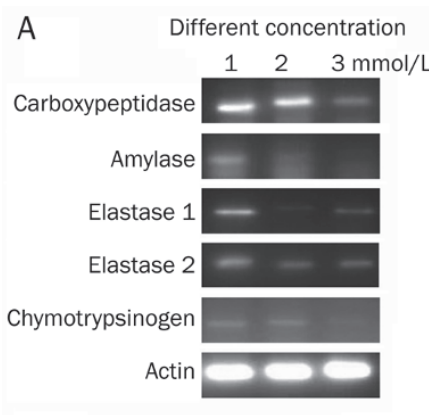

B

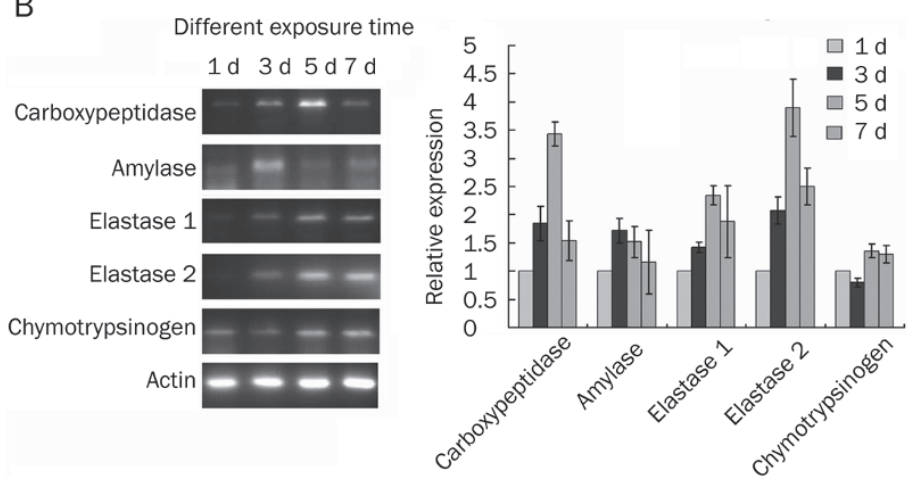

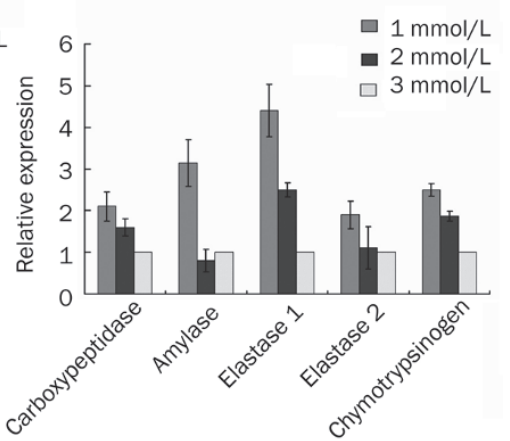

Figure 3. Sodium butyrate activates exocrine pancreatic differentiation of ES cells. (A) Cells were cultured in medium supplemented with 1, 2, or $3 \mathrm{mmol} / \mathrm{L}$ sodium butyrate for $5 \mathrm{~d}$. RTPCR was then performed to analyze exocrine pancreatic gene expression. (B) Cells were treated with $1 \mathrm{mmol} / \mathrm{L}$ sodium butyrate for 1 week. On d 1, 3, 5, and 7, cells were collected for analysis of the time course of exocrine gene expression. $\beta$-actin served as a control of equal RNA amount and the experiment was repeated three times. Histogram plots represent the densitometric analysis, corresponding to the means $\pm \mathrm{SD}$ of three independent experiments. 
terized in developing EBs. The results revealed that after $48 \mathrm{~h}$ of treatment, activinA induced upregulation of exocrine gene expression, whereas mRNA expression of exocrine genes was barely detectable in the absence of activinA (Figure 4A). To further compare gene expression in spontaneously differentiated and sodium butyrate-treated cells, EBs were exposed to $1 \mathrm{mmol} / \mathrm{L}$ sodium butyrate for $5 \mathrm{~d}$. The data indicated that 1 $\mathrm{mmol} / \mathrm{L}$ sodium butyrate significantly promoted higher exocrine gene expression than that of spontaneously differentiated cells. More specifically, the gene expression of amylase, elastase, and chymotrypsinogen increased by about 35\%,60\% and $35 \%$, respectively (Figure $4 \mathrm{~B}$ ).

Previous studies have shown that glucocorticoids influence
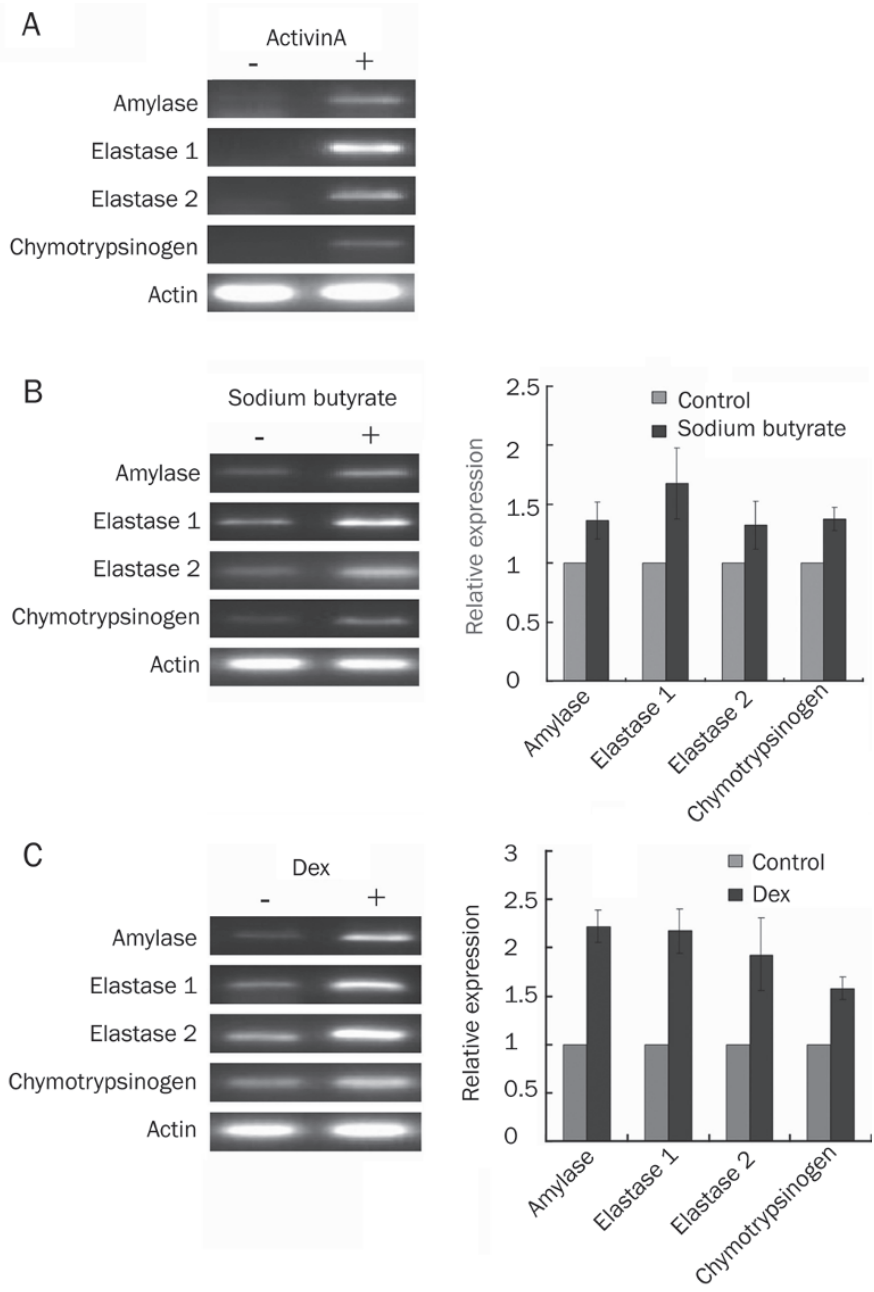

Figure 4. Induction of exocrine differentiation by activinA or Dex. EBs grown in suspension were cultured with $25 \mathrm{ng} / \mathrm{mL}$ activinA for $48 \mathrm{~h}(\mathrm{~A})$, $1 \mathrm{mmol} / \mathrm{L}$ sodium butyrate for 5 days (B), or $10^{-7} \mathrm{~mol} / \mathrm{L}$ Dex for seven days (C). RNA from spontaneously differentiated cells as well as treated cells was isolated and RT-PCR was performed to measure the mRNA levels of exocrine genes. The ratios of exocrine markers to $\beta$-actin were then calculated. Values are given as means $\pm S D$. For each experiment, a representative PCR result is shown. Histogram plots represent the densitometric analysis, corresponding to the means $\pm S D$ of three independent experiments. exocrine pancreas differentiation and increase exocrine gene expression $^{[17]}$. To assess the effect of the glucocorticoid Dex on ES cell exocrine differentiation, EBs were grown in Dex-containing medium for $7 \mathrm{~d}$. We found that Dex upregulated the gene expression of amylase, elastase, and chymotrypsinogen and exocrine gene expression in Dex-treated cells was about twofold higher than that of spontaneously differentiated cells (Figure 4C).

Enhancement of exocrine pancreatic differentiation by combining activinA, sodium butyrate, and Dex

Because a single inducing agent cannot achieve high differentiation efficiency, a combination of differentiation agents was needed to enhance exocrine pancreatic differentiation. To induce final differentiation, activinA was used for the first $2 \mathrm{~d}$, followed by the addition of sodium butyrate for $5 \mathrm{~d}$. Finally, following activinA and sodium butyrate treatment, the differentiated cells were transferred into Dex-containing medium for another $7 \mathrm{~d}$. Compared with activinA treatment alone, the combination of sodium butyrate and activinA resulted in higher levels of exocrine gene expression (Figure 5). Final treatment with Dex induced more marked amylase, elastase, and chymotrypsinogen gene expression compared with treatment with activinA and sodium butyrate alone. Meanwhile,

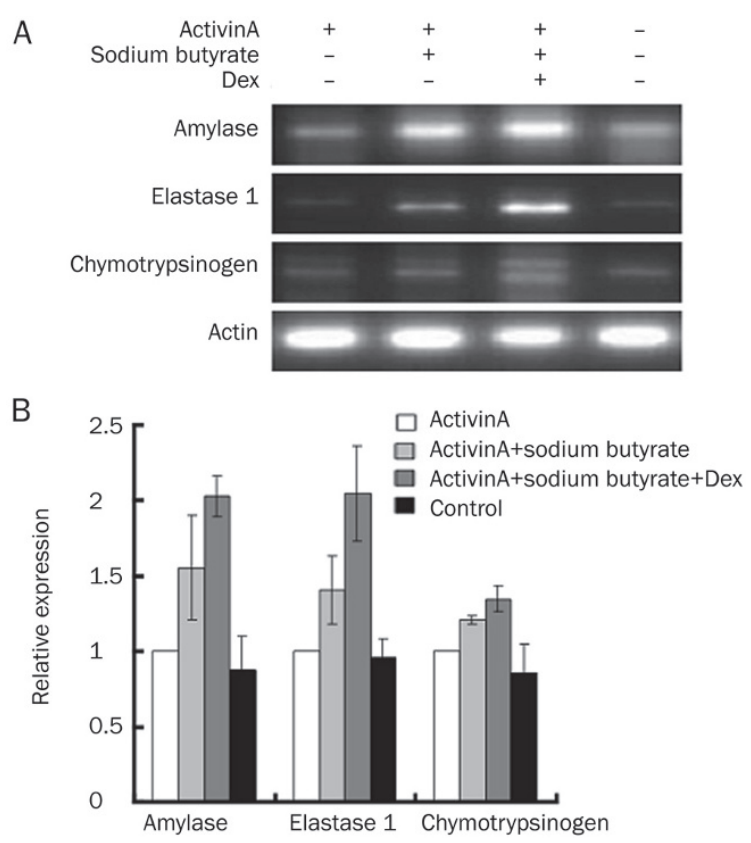

Figure 5. Expression of exocrine markers in EBs incubated with activinA, sodium butyrate and Dex. EBs grown in suspension were cultured in 25 $\mathrm{ng} / \mathrm{mL}$ activinA for $48 \mathrm{~h}$, followed by $1 \mathrm{mmol} / \mathrm{L}$ sodium butyrate treatment for 5 days. At the final stage of differentiation, $10^{-7} \mathrm{~mol} / \mathrm{L}$ Dex was added for another 7 days. RNA from both spontaneously differentiated and treated cells was isolated and RT-PCR was performed to measure the mRNA levels of exocrine genes. The ratios of exocrine markers to $\beta$-actin were calculated. Values are given as means $\pm S D$. (A) A representative PCR result is shown. (B) A histogram plot representing densitometric analysis, corresponding to the means $\pm \mathrm{SD}$ of three independent experiments. 
exocrine gene expression in spontaneously differentiated cells clearly decreased over the same 14-d culture duration (Figure 5).

At the final stage of differentiation, the spontaneously differentiated cells appeared irregularly shaped and fibroblastlike (Figure 6A). In contrast, treatment with inducing factors caused numerous cells with homogeneous morphology to spread from the EBs. These cells were polygonal with large, round, and center-situated nuclei (Figure 6B). Further immunofluorescence studies also revealed that some cells immunoreactive for amylase were located among the differentiated cells (Figure 6C). To measure the percentage of amylase-positive cells, we performed flow cytometry and found that approximately $17 \%$ of the differentiated cells were amylase-positive, which was higher than the percentage of spontaneously differentiated cells (Figure 6D).

\section{Discussion}

In the present study, we demonstrated that exposure to a low concentration of sodium butyrate promoted exocrine pancreatic differentiation in ES cells. Further combination with Dex and other inducing factors involved in pancreatic development significantly enhanced the differentiation efficiency.

The pancreas is a complex organ composed of both exocrine and endocrine regions. Acinar cells constitute $90 \%$ of the pancreatic epithelium and play a central role in pancreatic diseases, such as pancreatitis and pancreatic cancer. Although recent studies have indicated that ES cells can be induced to differentiate into insulin-secreting cells ${ }^{[7,8]}$, until now there have been only limited methods for studying acinar-cell differentiation in vitro. Because both the liver and the pancreas develop from the common embryonic endoderm and because these two lineages can transdifferentiate into each other ${ }^{[18,19]}$, exogenous factors that induce hepatic differentiation may also affect pancreatic differentiation. Our previous work and that of others have demonstrated that sodium butyrate, an inhibitor of histone deacetylase, can efficiently induce the differentiation of hepatocytes from ES cells ${ }^{[20,21]}$. Here, we investigated the influence of sodium butyrate on exocrine pancreatic differentiation.

First, we explored pancreas-specific gene expression in spontaneously differentiated ES cells before definitive induction into acinar cells. Because the presence of the three germ layers is important in the induction of definitive differentiation $^{[10]}$, the ES cells were cultured in suspension to form EBs containing endoderm, ectoderm, and mesoderm. Spontaneous EB differentiation included the generation of cells that shared characteristics with insulin-producing cells. Our results indicated that EBs can also express exocrine pancreatic markers when spontaneously differentiated, which is consistent with the results of Skoudy ${ }^{[22]}$. The coexistence of exocrine and endocrine cells in developing EBs suggests that local signals
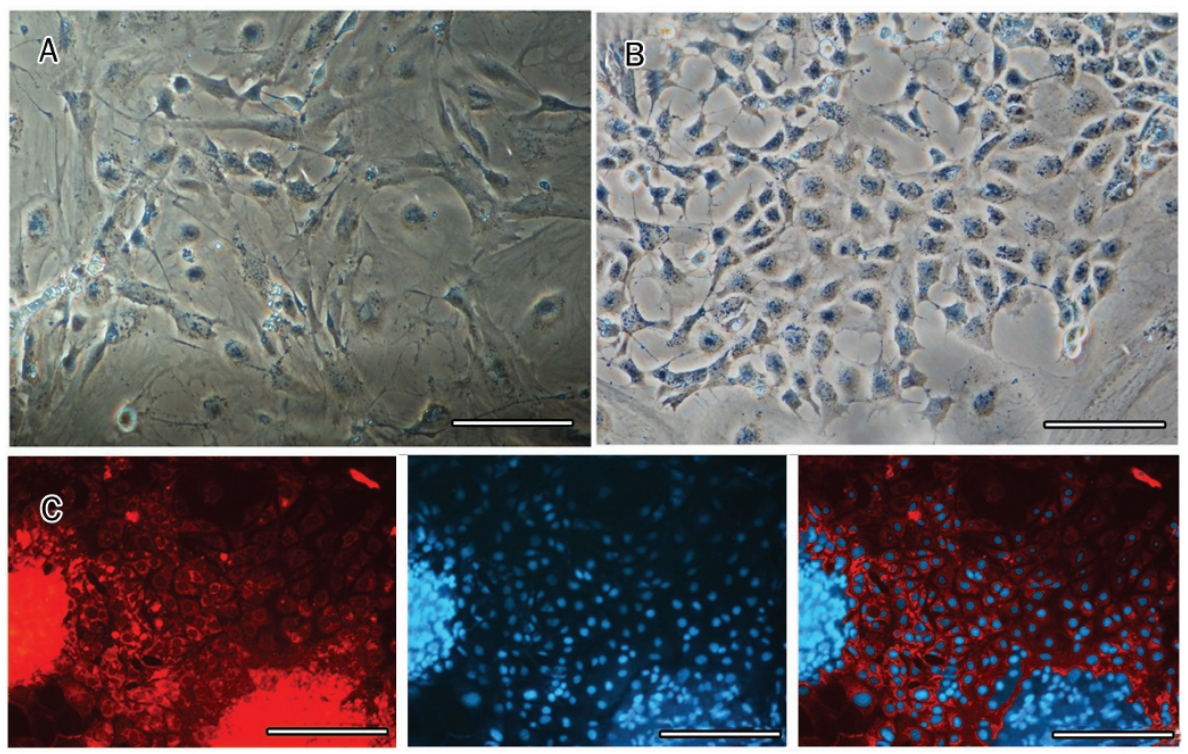

D

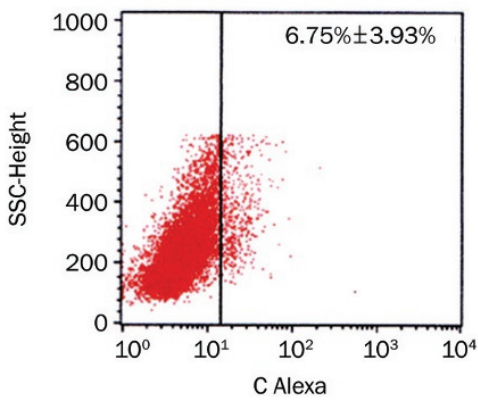

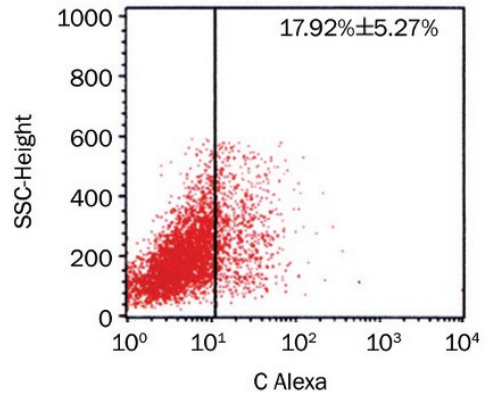

Figure 6. Change in cell morphology and amylase protein expression after exposure to activinA, sodium butyrate, and Dex. After the combined induction of differentiation using activinA, sodium butyrate, and Dex, cell morphology was observed by light microscopy. (A) Images of spontaneously differentiated cells serving as a control. (B) Images of differentiated cells. (C) Amylase was also detected in differentiated cells by immunofluorescence staining with antiamylase antibody. The red color depicts CY3conjugated amylase protein in the cytoplasm, whereas the blue color indicates the Hoechst 33258-stained nucleus. Representative images from three experiments performed on different days are shown. Scale bars: 50 $\mu \mathrm{m}$. (D) Flow cytometry analysis of amylase expression in spontaneously differentiated cells (left panel) and final differentiated cells (right panel). 
produced within the EBs are able to promote the exocrine and endocrine differentiation programs.

Sodium butyrate, a common dietary micronutrient, was an important component of our differentiation protocol. It has been widely used to differentiate ES cells into hepatocytes ${ }^{[20,21]}$ and has been found to induce up-regulation of PDX-1 and p48 in mouse ES cells upon brief exposure ${ }^{[12]}$. PDX-1, the earliest marker of pancreatic cells, and p48, which is crucial for exocrine cell differentiation, are both required for early pancreatic development. Here, we found that both PDX-1 and p48 were expressed at significantly higher levels in EBs treated with sodium butyrate. Exocrine enzyme gene expression was also promoted by sodium butyrate treatment, in contrast with untreated, spontaneously differentiated EBs. Moreover, we found that a relatively low dose of sodium butyrate for a short exposure time efficiently induced the up-regulation of genes associated with the exocrine pancreas. Although sodium butyrate is thought to act via histone deacetylation when inducing hepatocyte differentiation ${ }^{[21,23]}$, its mechanism of action during exocrine pancreas differentiation remains unclear.

Because a single factor could not efficiently induce ES cell differentiation, we investigated the effects of combining sodium butyrate and activinA on ES cell exocrine differentiation. ActivinA is a disulfide-stabilized protein that belongs to the TGF- $\beta$ superfamily. Its binding to a cell surface receptor can induce the expression of many genes important for early endoderm development such as Foxa2 and Sox17 $7^{[24]}$. Additionally, activinA improves insulin secretion by cultured human islets ${ }^{[25]}$ and regulates endocrine and exocrine differentiation during pancreatic development ${ }^{[26]}$. In the current study, we found the combination of sodium butyrate and activinA induced more marked exocrine gene expression than activinA treatment alone. One potential explanation for this finding is that sodium butyrate triggered epigenetic changes in the ES cells, making them more responsive to activinA-mediated gene expression.

Previous studies have shown that glucocorticoids influence exocrine pancreas differentiation and increase the expression of genes for amylase and other digestive enzymes ${ }^{[27]}$. In vitro treatment of the embryonic pancreas with Dex, a glucocorticoid agonist, decreased the number of insulin-expressing cells and doubled the acinar cell area, indicating that glucocorticoids favor acinar differentiation. Meanwhile, PDX-1 expression was downregulated and the mRNA levels for p48 were increased ${ }^{[28]}$. Dex has also been used to differentiate ES cells into hepatocytes, which, like pancreatic cells, are also derivatives of the definitive endoderm. In our study, Dex was added at the final stage of differentiation and then exocrine enzyme gene expression was tested. We found that exocrine gene expression increased after Dex treatment, in accordance with increased p48 expression. Thus, Dex may have profound effects on the exocrine differentiation of ES cells in vivo. However, the molecular mechanisms by which glucocorticoids mediate their effects have only been partly elucidated. Glucocorticoids may regulate the expression of specific genes, the products of which are important for the differentiated function of the acinar pancreas. The underlying signaling pathways involved in ES cell differentiation must be further investigated.

In summary, the present study demonstrates that sodium butyrate, an inhibitor of histone deacetylase, and the glucocorticoid Dex can promote exocrine pancreatic gene expression during ES cell differentiation. It was also found that a combination of these factors may increase induction efficiency. Thus, this study gives insight into pancreas formation and provides a model for the study of pancreas-related diseases.

\section{Acknowledgments}

This work was supported by grants from the China National Programs for High Technology Research and Development (2007AA02Z117), the National Natural Science Foundation of China (30571805), the Natural Science Foundation of Guang Dong province (8451008901000641) and the China Postdoctoral Science Foundation (20080430113).

\section{Author contribution}

Jun MIN and Hua CHENG designed research; Meng REN and Jun CAO performed research; Chang-zhen SHANG contributed new analytical tools and reagents; Fang-ping LI and Jingyi LI analyzed data; Meng REN, Li YAN and Chang-zhen SHANG wrote the paper.

\section{References}

1 Smith AG. Embryo-derived stem cells: of mice and men. Annu Rev Cell Dev Biol 2001; 17: 435-62.

2 Prelle K, Zink N, Wolf E. Pluripotent stem cells-model of embryonic development, tool for gene targeting, and basis of cell therapy. Anat Histol Embryol 2002; 31: 169-86.

3 Keller GM. In vitro differentiation of embryonic stem cells. Curr Opin Cell Biol 1995; 7: 862-9.

4 Klug MG, Soonpaa MH, Koh GY, Field LJ. Genetically selected cardiomyocytes from differentiating embronic stem cells form stable intracardiac grafts. J Clin Invest 1996; 98: 216-24.

5 Yamada T, Yoshikawa M, Kanda S, Kato Y, Nakajima Y, Ishizaka S, et al. In vitro differentiation of embryonic stem cells into hepatocyte-like cells identified by cellular uptake of indocyanine green. Stem Cells 2002; 20: 146-54.

6 Ibii T, Shimada H, Miura S, Fukuma E, Sato H, Iwata H. Possibility of insulin-producing cells derived from mouse embryonic stem cells for diabetes treatment. J Biosci Bioeng 2007; 103: 140-6.

7 Ku HT, Chai J, Kim YJ, White P, Purohit-Ghelani S, Kaestner KH, et al. Insulin-expressing colonies developed from murine embryonic stem cell-derived progenitors. Diabetes 2007; 56: 921-9.

8 Jiang J, Au M, Lu K, Eshpeter A, Korbutt G, Fisk G, et al. Generation of insulin-producing islet-like clusters from human embryonic stem cells. Stem Cells 2007; 25: 1940-53.

9 Blouin MJ, Lamy I, Loranger A, Noel M, Corlu A, Guguen-Guillouzo C, et al. Specialization switch in differentiating embryonic rat liver progenitor cells in response to sodium butyrate. Exp Cell Res 1995; 217 : 22-30.

10 Rambhatla L, Chiu CP, Kundu P, Peng Y, Carpenter MK. Generation of hepatocyte-like cells from human embryonic stem cells. Cell Transplant 2003; 12: 1-11. 
11 McKiernan E, O'Driscoll L, Kasper M, Barron N, O'Sullivan F, Clynes M. Directed differentiation of mouse embryonic stem cells into pancreatic-like or neuronal- and glial-like phenotypes. Tissue Eng 2007; 13: 2419-30.

12 Goicoa S, Alvarez S, Ricordi C, Inverardi L, Dominguez-Bendala J. Sodium butyrate activates genes of early pancreatic development in embryonic stem cells. Cloning Stem Cells 2006; 8: 140-9.

13 Yamada S, Terada K, Ueno Y, Sugiyama T, Seno M, Kojima I. Differentiation of adult hepatic stem-like cells into pancreatic endocrine cells. Cell Transplant 2005; 14: 647-53.

14 Krapp A, Knofler M, Ledermann B, Burki K, Berney C, Zoerkler N, et al. The bHLH protein PTF1-p48 is essential for the formation of the exocrine and the correct spatial organization of the endocrine pancreas. Genes Dev 1998; 12: 3752-63.

15 Gittes GK, Rutter WJ. Onset of cell-specific gene expression in the developing mouse pancreas. Proc Natl Acad Sci USA 1992; 89: 1128-32.

16 Kim SK, Hebrok M, Li E, Oh SP, Schrewe H, Harmon EB, et al. Activin receptor patterning of foregut organogenesis. Genes Dev 2000; 14 : 1866-71.

17 Rall L, Pictet R, Githens S, Rutter WJ. Glucocorticoids modulate the in vitro development of the embryonic rat pancreas. J Cell Biol 1977; 75: 398-409.

18 Zaret KS, Grompe M. Generation and regeneration of cells of the liver and pancreas. Science 2008; 322: 1490-4.

19 Soto-Gutierrez A, Navarro-Alvarez N, Caballero-Corbalan J, Tanaka N, Kobayashi N. Endoderm induction for hepatic and pancreatic differentiation of ES cells. Acta Med Okayama 2008; 62: 63-8.

20 Min J, Shang CZ, Chen YJ, Zhang L, Liu L, Deng XG, et al. Selective enrichment of hepatocytes from mouse embryonic stem cells with a culture system containing cholestatic serum. Acta Pharmacol Sin 2007; 28: 1931-7.

21 Zhou QJ, Xiang LX, Shao JZ, Hu RZ, Lu YL, Yao H, et al. In vitro differentiation of hepatic progenitor cells from mouse embryonic stem cells induced by sodium butyrate. J Cell Biochem 2007; 100: 29-42.

22 Skoudy A, Rovira M, Savatier P, Martin F, Leon-Quinto T, Soria B, et al. Transforming growth factor (TGF)beta, fibroblast growth factor (FGF) and retinoid signalling pathways promote pancreatic exocrine gene expression in mouse embryonic stem cells. Biochem J 2004; 379: 749-56.

23 Sharma NS, Shikhanovich R, Schloss R, Yarmush ML. Sodium butyrate-treated embryonic stem cells yield hepatocyte-like cells expressing a glycolytic phenotype. Biotechnol Bioeng 2006; 94: 1053-63.

24 Parashurama N, Nahmias $\mathrm{Y}$, Cho $\mathrm{CH}$, van Poll D, Tilles AW, Berthiaume $\mathrm{F}$, et al. Activin alters the kinetics of endoderm induction in embryonic stem cells cultured on collagen gels. Stem Cells 2008; 26: 474-84.

25 Demeterco C, Beattie GM, Dib SA, Lopez AD, Hayek A. A role for activin $A$ and betacellulin in human fetal pancreatic cell differentiation and growth. J Clin Endocrinol Metab 2000; 85: 3892-7.

26 Nakanishi M, Hamazaki TS, Komazaki S, Okochi H, Asashima M. Pancreatic tissue formation from murine embryonic stem cells in vitro. Differentiation 2007; 75: 1-11.

27 Logsdon CD, Moessner J, Williams JA, Goldfine ID. Glucocorticoids increase amylase mRNA levels, secretory organelles, and secretion in pancreatic acinar AR42J cells. J Cell Biol 1985; 100: 1200-8.

28 Gesina E, Tronche F, Herrera P, Duchene B, Tales W, Czernichow P, et al. Dissecting the role of glucocorticoids on pancreas development. Diabetes 2004; 53: 2322-9. 